\title{
Non-equilibrium phase transition in a two-species driven-diffusive model of classical particles
}

\author{
Mohammad Ghadermazi $^{1}$ - Farhad H. Jafarpour ${ }^{2}$
}

Received: 23 January 2016/ Accepted: 22 March 2016/Published online: 27 June 2016

(c) The Author(s) 2016. This article is published with open access at Springerlink.com

\begin{abstract}
A two-species driven-diffusive model of classical particles is introduced on a lattice with periodic boundary condition. The model consists of a finite number of first class particles in the presence of a second class particle. While the first class particles can only hop forward, the second class particle is able to hop both forward and backward with specific rates. We have shown that the partition function of this model can be calculated exactly. The model undergoes a non-equilibrium phase transition when a condensation of the first class particles occurs behind the second class particle. The phase transition point and the spatial correlations between the first class particles are calculated exactly. On the other hand, we have shown that this model can be mapped onto a two-dimensional walk model. The random walker can only move on the first quarter of a two-dimensional plane and that it takes the paths which can start at any height and end at any height upper than the height of the starting point. The initial vertex (starting point) and the final vertex (end point) of each lattice path are weighted. The weight of the outset point depends on the height of that point while the weight of the end point depends on the height of both the outset point and the end point of each path. The partition function of this walk model is calculated using a transfer matrix method.
\end{abstract}

Mohammad Ghadermazi

m.ghadermazi@iausdj.ac.ir

1 Plasma Physics Research Center, Science and Research Branch, Islamic Azad University, Tehran, Iran

2 Physics Department, Bu-Ali Sina University, 65174-4161 Hamedan, Iran
Keywords Driven-diffusive model · Walk model · Correlation function $\cdot$ Zero-range processes

\section{Introduction}

One of the most studied models which shows non-equilibrium phase transitions is asymmetric simple exclusion process (ASEP). In many literatures, the ASEP in the presence of an impurity on a ring have been studied. The role of the impurity is to investigate the motion of the shock fronts in the ASEP. The single impurity in $[1,2]$ hops in the opposite direction relative to the ordinary particle of the ASEP while in [3] the impurity moves in the same direction as the ordinary particles of the ASEP. In both cases the phase structure of the models have been studied extensively.

In this paper, we study the effects of the presence of a single impurity on the ASEP on a ring where the second class particle (impurity) is allowed to hop in the both directions, relative to the ordinary particle of the ASEP, with the rates $q$ and $p$. It has been shown that the steadystate distribution of all one-dimensional exclusion models whose steady-states have a simple factorized form, can be written in a matrix product form. The matrices which are necessary for this purpose satisfy a generalized quadratic algebra [4]. In [5], the authors have introduced a mathematical tool for studying of correlations in the models whose steady-states have a simple factorized form. They use the matrices which satisfy a generalized quadratic algebra. In this paper, we introduce an infinite-dimensional matrix representation which satisfies the quadratic algebra of the model. The canonical partition function of the model is calculated exactly. Using a canonical ensemble, the phase structure of the model is studied in thermodynamic 
limit. The steady-state distribution of our model has a factorized form hence two matrix representations are presented which satisfy the generalized quadratic algebra of the model. An infinite-dimensional matrix representation and a 2-dimensional matrix representation. Using this matrix representations the calculations seem to be very straightforward. It has been shown that using the grand canonical partition function one can analyze the phase structure of the model. The transition point can be calculated numerically or analytically [6, 7]. By assigning the fugacity $z$ to the first class particles in a grand canonical ensemble, we shall find the exact phase structure and calculate the density profile and the correlations of the first class particles precisely. Some of the critical exponents of the model in phase transition is also obtained.

In recent years, many studies have been done on connections between the one-dimensional driven-diffusive systems and the two-dimensional walk models [8-10]. It has been shown that the partition function of some of the onedimensional driven-diffusive models with open boundaries obtained using a matrix product method is equal to the partition function of a two-dimensional walk model obtained using a transfer matrix method [8, 11, 12]. In [11], the author has introduced a lattice path with specific dynamical rules where walker can start from origin and end at any height upper than origin and it has been shown that the partition function of this two-dimensional walk model is exactly equal to that of a driven-diffusive system defined on a discrete lattice with periodic boundary conditions that can be mapped to a zero-range process $[13,14]$. In this paper, a two-dimensional walk model is introduced in which the walker can only move on the first quarter of a two-dimensional plane. Random walker can start moving at any height upper than the origin and end at any height upper than the starting point. All the paths made by the random walker are weighted. The weight of a given path will be equal to the product of the weights of the consecutive steps in that path and the weight of the starting and end points. The partition function of this walk model is the sum of the unnormalized weights of different paths consisting of $t-1$ steps and is calculated using a transfer matrix method.

The paper is organized as follows: In "The driven-diffusive model" a two-species driven-diffusive model is introduced. In "The canonical partition function" the canonical partition function of the model is calculated in the thermodynamic limit and the phase structure of the model is investigated. In "The spatial correlations" the correlation functions and the critical exponent of the model are calculated. In "The walk model" we introduce a two-dimensional walk model related to the two-species driven-diffusive model.

\section{The driven-diffusive model}

In [11], the author has introduced a one-dimensional driven-diffusive model of classical particles with hardcore interactions. The model consists of a single particle of type $A$ (called the second-class particle) and $M-1$ particles of type $B$ (called the first-class particles). The particles move on a one-dimensional lattice of length $t$ with periodic boundary condition. The particle of type $A$ hops from the lattice site $i$ to $i+1$ with the rate $p$ provided that the target site is empty. A particle of type $B$ hops from the lattice site $i$ to $i+1$ with the rate 1 provided that the target site is empty.

In this paper, we assume that the second-class particle is also allowed to hop backward with the rate $q$. If an empty lattice site is denoted by $\emptyset$, we can summarize the reaction rules at a pair of lattice sites $i$ and $i+1$ as follows
$A \emptyset \rightarrow \emptyset A$ with rate $p$
$\emptyset A \rightarrow A \emptyset \quad$ with rate $q$
$B \emptyset \rightarrow \emptyset B \quad$ with rate 1 .

In the long-time limit the system attains a non-equilibrium steady-state. It can be shown that the probability distribution can be obtained using a matrix product method. For this purpose, we label the particle of type $A$ with 1 and label the particles of type $B$ with $2,3, \ldots, M$. If the number of empty lattice sites in front of the $i$ 'th particle is denoted by $n_{i}$, a general configuration of the model can be written as $\{n\}=\left\{n_{1}, n_{2}, \ldots, n_{M}\right\}$. If the lattice site is occupied by the particle of type $A$, the matrix $D_{1}$ is attributed to it. If a lattice site is occupied by a particle of type $B$, the matrix $D_{2}$ is attributed to it. In the steady-state the probability of finding the system in a general configuration $\{n\}=$ $\left\{n_{1}, n_{2}, \ldots, n_{M}\right\}$ is given by

$P(\{n\})=\frac{1}{Z_{t, M}(p, q)} \operatorname{Tr}\left(D_{1} E^{n_{1}} D_{2} E^{n_{2}} \ldots D_{M} E^{n_{M}}\right)$

in which $Z_{t, M}(p, q)$ is the normalization factor which is also called the canonical partition function of the model and that it should be calculated by considering the conservation of the number of empty sites i.e., $\sum_{v=1}^{M} n_{v}=t-M$. A sufficient condition for (1) to be the steady-state probability distribution of the model is

$p D_{1} E-q E D_{1}=D_{1}$

$D_{2} E=D_{2}$.

Details of the proof is given in [15]. It can easily be verified that the above algebra has the following infinite-dimensional matrix representation 


$$
\begin{aligned}
D_{1} & =\sum_{i=0}^{\infty} \sum_{i^{\prime}=i}^{\infty}\left(\begin{array}{c}
i^{\prime} \\
i
\end{array}\right) \frac{q^{i}}{p^{i^{\prime}}}|i\rangle\left\langle i^{\prime}\right|, \\
D_{2} & =\sum_{i=0}^{\infty}|0\rangle\langle i|, \\
E & =\sum_{i=0}^{\infty}|i+1\rangle\langle i|
\end{aligned}
$$

in which $|i\rangle_{j}=\delta_{i, j}$ for $i, j=0,1, \ldots, \infty$.

\section{The canonical partition function}

The number of empty lattice sites is a conserved quantity and does not change by the dynamical rules; therefore, using (1) one can calculate the canonical partition function of the model as follow

$$
Z_{t, M}(p, q)=\sum_{n_{1}, \ldots, n_{M}=0}^{t-M} \operatorname{Tr}\left[D_{1} E^{n_{1}} D_{2} E^{n_{2}} \ldots D_{2} E^{n_{M}}\right] \delta_{\sum_{v=1}^{M} n_{v},(t-M)} .
$$

Using the matrix representations of $D_{2}$ and $E$, one can write

$$
D_{2} E^{n}=D_{2} \text {. }
$$

Using the above equation we can rewrite the canonical partition function of the model as follows

$$
Z_{t, M}(p, q)=\sum_{n_{1}, \ldots, n_{M}=0}^{t-M} \operatorname{Tr}\left[D_{1} E^{n_{1}} D_{2}\right] \delta_{\sum_{v=1}^{M} n_{v},(t-M)} .
$$

Using the matrix representation of the matrices $D_{1}, D_{2}$ and $E$ and the definition of trace of a matrix one can obtain

$$
\begin{aligned}
\operatorname{Tr}\left[D_{1} E^{n_{1}} D_{2}\right] & =\sum_{i=0}^{\infty}\left\langle i\left|D_{1} E^{n_{1}} D_{2}\right| i\right\rangle \\
& =\sum_{i=0}^{n_{1}}\left(\begin{array}{c}
n_{1} \\
i
\end{array}\right) \frac{q^{i}}{p^{n_{1}}} .
\end{aligned}
$$

Inserting the above equation into (4), the canonical partition function of the model can be written as

$$
Z_{t, M}(p, q)=\sum_{n_{1}=0}^{t-M} \sum_{i=0}^{n_{1}}\left(\begin{array}{c}
n_{1} \\
i
\end{array}\right)\left(\begin{array}{c}
t-n_{1}-2 \\
M-2
\end{array}\right) \frac{q^{i}}{p^{n_{1}}} .
$$

It can be seen that the partition function of the model in thermodynamic limit $M, t \rightarrow \infty$ behaves as

$$
Z_{t, M}(p, q) \simeq\left\{\begin{array}{cl}
p^{t(\rho-1)}(1+q)^{t-1}(1+q-p)^{2-M} & \text { for } \rho<\frac{1-p+q}{1+q} \\
\left(\begin{array}{c}
t \\
M
\end{array}\right) \frac{p \rho^{2}}{p-(1-\rho)(1+q)} & \text { for } \rho>\frac{1-p+q}{1+q}
\end{array}\right.
$$

in which $\rho$ is the density of the first-class particle and is given by $\rho=(M-1) / t \simeq M / t$. It can be seen that a phase transition occurs at $\rho=(1-p+q) /(1+q)$. To investigate the phase behavior of the model we calculate the mean number of the empty lattice sites in front of the second-class particle. Given that the total number of empty lattice sites on the lattice is $t-M$, the probability that the number of empty lattice sites in front of the second-class particle is $n_{1}$, is given by

$$
P_{t, M}\left(n_{1}\right)=\frac{1}{Z_{t, M}(p, q)} \sum_{i=0}^{n_{1}} \frac{q^{i}}{p^{n_{1}}}\left(\begin{array}{c}
n_{1} \\
i
\end{array}\right)\left(\begin{array}{c}
t-n_{1}-2 \\
M-2
\end{array}\right)
$$

Hence the average number of empty lattice sites in front of the particle of type $A$ is

$\bar{n}_{1}=\sum_{n_{1}=0}^{t-M} n_{1} P_{t, M}\left(n_{1}\right)=-p \frac{\partial \ln Z_{t, M}(p, q)}{\partial p}$.

Using (6), the average number of empty lattice sites in front of the particle of second-class particle in the thermodynamic limit is as follows

$$
\bar{n}_{1}=\left\{\begin{array}{ll}
t\left(1-\rho-\frac{p \rho}{1+q-p}\right) & \text { for } \rho<\frac{1-p+q}{1+q} \\
\frac{p}{p-(1-\rho)(1+q)}-1 & \text { for } \rho>\frac{1-p+q}{1+q}
\end{array} .\right.
$$

As can be seen there is a phase transition from a phase in which the mean number of empty lattice site in front of the second-class particle is of order $t$ to another phase where it is a constant.

\section{The spatial correlations}

In [4] the author has shown that the steady state of a disordered driven-diffusive system consisting of $M$ different type of particles, that can be mapped onto the Zero Range Process, can be obtained using the matrix method in which the matrices should satisfy the following generalized quadratic algebra

$$
D_{\mu} E^{n_{\mu}} D_{\mu^{\prime}}=f_{\mu}\left(n_{\mu}\right) D_{\mu^{\prime}} \quad \text { for } \mu, \mu^{\prime}=1,2, \ldots, M
$$

in which $f_{\mu}\left(n_{\mu}\right)$ is a function of transition rates and can be constructed using pairwise balance condition [16]. Our model is a two-species driven-diffusive model of classical particles on a lattice with periodic boundary condition with the following dynamic

$$
\begin{aligned}
& \mu^{\prime} \underbrace{0 \cdots 0}_{n_{\mu^{\prime}}} \mu \underbrace{0 \cdots 0}_{n_{\mu}} \mu^{\prime \prime} \rightarrow \mu^{\prime} \underbrace{0 \cdots 0}_{n_{\mu^{\prime}+1} \cdots 0} \mu \underbrace{0 \cdots 0}_{n_{\mu}-1} \mu^{\prime \prime} \quad \text { with the rate } u_{\mu}\left(n_{\mu}\right) \\
& \mu^{\prime} \underbrace{0 \cdots 0}_{n_{\mu^{\prime}}} \mu \underbrace{0 \cdots 0}_{n_{\mu}} \mu^{\prime} \rightarrow \mu^{\prime} \underbrace{0 \cdots 0}_{n_{\mu^{\prime}}-1} \mu \underbrace{0 \cdots 0}_{n_{\mu}+1} \mu^{\prime \prime} \quad \text { with the rate } v_{\mu}\left(n_{\mu}\right)
\end{aligned}
$$


where $u_{\mu}\left(n_{\mu}\right)$ is the hopping rate of the particle $\mu$ to its right neighboring lattice site and $v_{\mu}\left(n_{\mu}\right)$ is the hopping rate of the particle $\mu$ to its left neighboring lattice site i.e.,

$u_{1}\left(n_{1}\right)=p, \quad v_{1}\left(n_{1}\right)=q$,

$u_{2}\left(n_{2}\right)=1, \quad v_{2}\left(n_{2}\right)=0$.

It can be checked that by defining $f_{1}\left(n_{1}\right)=\left(\frac{1+q}{p}\right)^{n_{1}}$ and $f_{2}\left(n_{2}\right)=1$ and requiring $f_{1,2}(0)=1$, the following infinitedimensional matrix representation satisfies the quadratic algebra (10)

$$
\begin{aligned}
D_{1} & =\sum_{i=0}^{\infty} f_{1}(i)|0\rangle\langle i|, \\
D_{2} & =\sum_{i=0}^{\infty}|0\rangle\langle i|, \\
E & =\sum_{i=0}^{\infty}|i+1\rangle\langle i| .
\end{aligned}
$$

Using (13) the grand-canonical partition function of the model can be written as

$$
Z_{t}(p, q, z)=\operatorname{Tr}\left[D_{1} C^{t-1}\right]=\sum_{i=0}^{\infty} f(i)\left\langle i\left|C^{t-1}\right| 0\right\rangle
$$

where the matrix $C=E+z D_{2}$ and that $z$ is the fugacity of the first-class particles. According to the matrix representations (13) it can be verified that

$$
C^{t-1}|j\rangle=\sum_{i=0}^{t-2} z(z+1)^{t-i-2}|i\rangle+|j+t-1\rangle .
$$

Now the grand-canonical partition function $Z_{t}(p, q, z)$ can be calculated using (15)

$Z_{t}(p, q, z)=\frac{p z\left(\frac{1+q}{p}\right)^{t-1}}{1+q-p(1+z)}-\frac{p z(1+z)^{t-1}}{1+q-p(1+z)}+\left(\frac{1+q}{p}\right)^{t-1}$.

The fugacity $z$ has to be fixed by density of the first-class particles which is given by the following equation

$$
\rho(z)=\frac{z}{t} \frac{\partial}{\partial z} \ln Z_{t}(p, q, z) .
$$

It is known that the real positive values of the fugacity are of physical interest hence it is necessary that $p<1+q$. Using (16) it can be shown that in the thermodynamic limit the density of first-class particles can be written as follows

$$
\rho(z)=\left\{\begin{array}{cl}
\frac{z}{1+z} & \text { for } z>\frac{1+q-p}{p} \\
0 & \text { for } z<\frac{1+q-p}{p}
\end{array} .\right.
$$

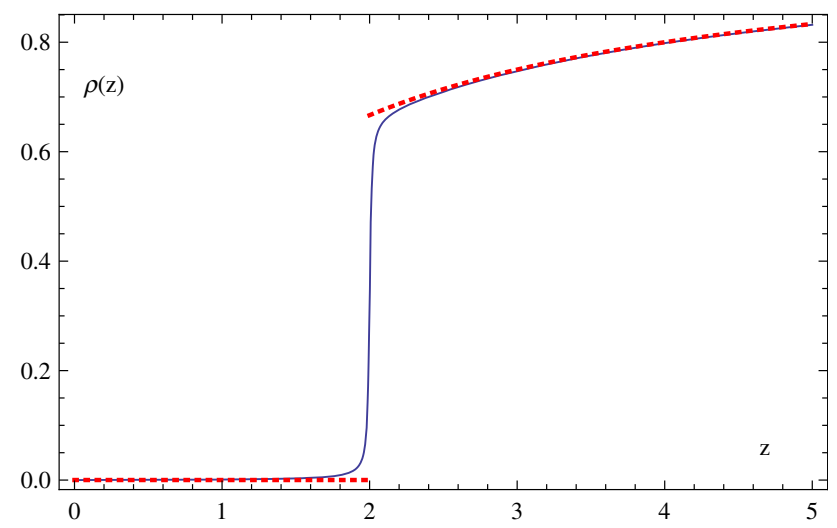

Fig. 1 The density of the first-class particles as a function of fugacity $z$ obtained from the exact solution (the blue line) and in the thermodynamic limit (the red dotted line) for $t=200, p=1$ and $q=2$

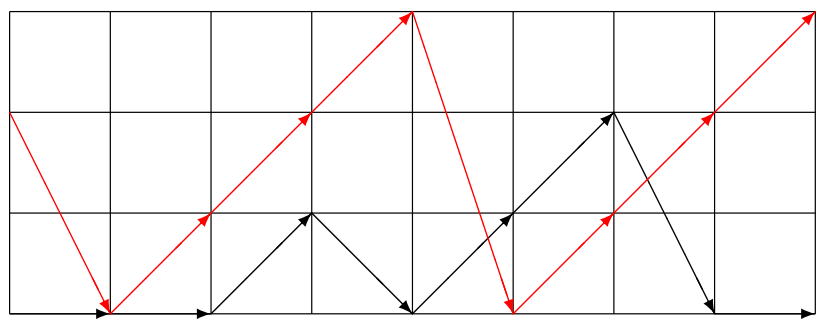

Fig. 2 Two lattice path that start from $(0,0)$ and $(0,2)$

According to (18) it turns out that there is a critical fugacity $z_{c}=\frac{1+q-p}{p}$ at which the density of the first-class particles shows a finite discontinuity. The behavior of $\rho(z)$ for $z<z_{c}$ and $z>z_{c}$ are different and the system undergoes a firstorder phase transition provided that $p<1+q$. In Figs. 1 and 2 exact expression of the density of the first-class particles and its thermodynamic limit are plotted as a function of the fugacity $z$. As can be seen, in the thermodynamic limit both plots overlap. At $z_{c}$ there is a finite discontinuity while for $z>z_{c}, \rho(z)$ grows with $z$ until it saturates.

In [5] the authors have studied the spatial correlations in exclusion models corresponding to the Zero Range Processes. They have shown that the spatial correlations of the exclusion models that can be mapped onto the Zero Range Processes can be expressed in terms of 1-point and 2-point correlation functions $G_{i}^{(1)}$ and $G_{i, j}^{(2)}$. Given that the only impurity is at site 1 , the density of the first-class particles at the lattice site $i$ can be written as

$$
G_{i}^{(1)}=\left\langle\rho_{i}\right\rangle=\frac{1}{Z_{t}(p, q, z)} \operatorname{Tr}\left[D_{1} C^{i-2}\left(z D_{2}\right) C^{t-i}\right] .
$$

Calculating (19) using (15) is straightforward and the result is 


$$
\left\langle\rho_{i}\right\rangle=A_{1}+A_{2} \exp \left(\frac{-i}{\xi}\right)
$$

in which $\xi$ is a correlation length which is given by

$$
\xi^{-1}=\ln \frac{p(1+z)}{1+q} .
$$

The coefficients $A_{1}$ and $A_{2}$ in (20) are functions of the transition rates $p, q$ and also the system size $t$

$$
\begin{aligned}
& A_{1}=\frac{p z^{2}(1+z)^{t-2}}{p z\left[(1+z)^{t-1}-\left(\frac{1+q}{p}\right)^{t-1}\right]+(1+q-p-p z)\left(\frac{1+q}{p}\right)^{t-1}}, \\
& A_{2}=\frac{z\left(\frac{1+q}{p}\right)^{-2}(1+q-p)(1+z)^{t}}{p\left(\frac{1+q}{p}\right)^{2} z\left[(1+z)^{t-1}-\left(\frac{1+q}{p}\right)^{t-1}\right]+(1+q-p-p z)\left(\frac{1+q}{p}\right)^{t-1}} .
\end{aligned}
$$

The density of the first class particles increases exponentially from the vicinity of the second-class particle. In the thermodynamic limit the density of the first class particles $\left\langle\rho_{i}\right\rangle$ behaves as (18) far from the second class particle.

It should be noted that in addition to infinite dimensional matrix representation (13) the quadratic algebra (10) has a 2-dimensional matrix representation. In [17] the authors have shown that the quadratic algebra (10) has a finitedimensional representation which depends on the number of types of particles. The dimension of the matrix is $M$ if the number of types of the particles is equal to $M$. Hence for our model with two species of particles the quadratic algebra (10) has a 2-dimensional matrix representation given by

$D_{1}=\left(\begin{array}{ll}1 & 0 \\ 1 & 0\end{array}\right) \quad, \quad D_{2}=\left(\begin{array}{ll}0 & 1 \\ 0 & 1\end{array}\right) \quad, \quad E=\left(\begin{array}{cc}\frac{1+q}{p} & 0 \\ 0 & 1\end{array}\right)$.

According to (23) the matrix $C=E+z D_{2}$ in (14) can be written as

$C=\left(\begin{array}{cc}\frac{1+q}{p} & z \\ 0 & 1+z\end{array}\right)$

It can be seen that the correlation length (21) can be written as a function of the eigenvalues of the 2-dimensional matrix $C$ as

$$
\xi^{-1}=\ln \left(\frac{\lambda_{1}}{\lambda_{2}}\right)
$$

where $\lambda_{1}=1+z$ and $\lambda_{2}=\frac{1+q}{p}$, in agreement with the known results obtained in [18]. The 2-point correlation function $G_{i, j}^{(2)}(z)=\left\langle\rho_{i} \rho_{j}\right\rangle$ can be written as
$G_{i, j}^{(2)}(z)=\frac{z^{2}}{Z_{t}(p, q, z)} \operatorname{Tr}\left[D_{1} C^{i-2} D_{2} C^{j-i-1} D_{2} C^{t-j}\right]$.

Using (15) and (20) and after some straightforward calculations one can obtain $G_{i, j}^{(2)}(z)$ explicitly

$G_{i, j}^{(2)}(z)=\frac{z}{1+z}\left\langle\rho_{i}\right\rangle$.

The $(n+1)$-point correlations are written as $G_{j_{1} \ldots j_{n}}^{(n+1)}=\left\langle\rho_{i} \rho_{i+j_{1}} \ldots \rho_{i+j_{1}+\cdots+j_{n}}\right\rangle$

$G_{j_{1} \ldots j_{n}}^{(n+1)}=\frac{1}{Z_{t}(p, q, z)} \operatorname{Tr}\left[D_{1} C^{i-2}\left(z D_{2}\right) C^{j_{1}-i-1}\left(z D_{2}\right) C^{j_{2}-j_{1}-1}\left(z D_{2}\right) \ldots C^{t-n}\right]$.

Using (19) and (26) we can express the above equation in terms of $G_{i}^{(1)}$ as follows

$G_{j_{1} \ldots j_{n}}^{(n+1)}=\left(\frac{z}{1+z}\right)^{n}\left\langle\rho_{i}\right\rangle$.

We can calculate the critical exponents of model at the phase transition point. To find the critical exponent defined by $\rho \propto\left(z-z_{c}\right)^{\beta}$, we only need to consider the behavior of the density of the first-class particles as a function of fugacity $z$ at the critical point $z_{c}=\frac{1+q-p}{p}$ in the thermodynamic limit. According to (17), it can be seen that the density of the first-class particles in the vicinity of $z_{c}$ can be expressed as $\rho \propto\left(z-z_{c}\right)^{-1}$. Hence the critical exponent $\beta=-1$. Near the critical fugacity, $(1+z) \rightarrow \frac{1+q}{p}$. According to (20), it can be seen that in the thermodynamic limit the density profile $\left\langle\rho_{i}\right\rangle \propto\left(z-z_{c}\right)^{-1}$. Hence, the critical exponent $\alpha$ defined by $\left\langle\rho_{i}\right\rangle \propto\left(z-z_{c}\right)^{\alpha}$ is $\alpha=-1$. With the correlation function given asymptotically by $G_{i, j}^{(2)} \sim(j-i)^{-D+2-\eta} \exp \left(\frac{-(j-i)}{\xi}\right)$ in which $D$ is the dimension of the system, we find $\eta=1$.

\section{The walk model}

In this section, we show that there exists a walk model which is equivalent to the driven-diffusive model explained in the previous sections. We consider a two-dimensional walk model in which a random walker can start from any height upper than the origin $(0, j)$ in which $j$ is an integer $j \geq 0$. We assume that the random walker can take a finite number of steps on $\mathrm{Z}_{+}^{2}=\{(i, j): i, j \geq 0 \quad$ are integers $\}$ according to the rules which will be explained later. For the reasons that will become clear shortly we assume that the length of the lattice path is equal to $t-1$. After taking a finite number $t-1$ of consecutive steps, the random walker can get to the lattice site $\left(t-1, j^{\prime}\right)$ where $j^{\prime}=j, j+1, \ldots, j+t-1$. The initial vertex (starting point) and the final vertex (end point) 
of the lattice path are weighted. This type of lattice path is introduced in [19]. For any path the weight of the start and end points depend on the height of these points. There are different ways that after taking the finite number of steps $t-1$, the random walker can get to the lattice site $\left(t-1, j^{\prime}\right)$. The weight of a given path will be equal to the product of the weights of the start and end points and the consecutive steps. The random walker moves according to the following rules:

1. The random walker can start from any height upper than the origin as $(0, j)$ where $j=0,1,2, \ldots, \infty$.

2. A path that starts from the height $(0, j)$, after $t-1$ steps might terminate at any height such as $\left(t-1, j^{\prime}\right)$ where $j^{\prime}=j, j+1, j+2, \ldots, j+t-1$.

3. The weight of the initial vertex (starting point) for the path that starts from the height $(0, j)$ is $q^{j}$.

4. The weight of the final vertex (end point) for the path that starts from the height $(0, j)$ and terminates to the lattice site $\left(t-1, j^{\prime}\right)$, is $\left(\begin{array}{c}j^{\prime} \\ j\end{array}\right) \frac{1}{p^{\prime}}$

5. For $i \geq j$ and from the lattice site $(i, j)$ to $(i+1, j+1)$ the steps have the weight 1(upward steps).

6. For $i \geq j$ and from the lattice site $(i, j)$ the random walker can drop to the surface $(i+1,0)$. These steps have the weight 1 (jump steps for $j>0$ and horizontal steps for $j=0$ ).

In Fig. 2 we have plotted two different paths of length 8 according to the above mentioned rules. We will be interested in those paths of fixed length which contain a certain number of jumps and horizontal steps (equivalently upward steps); therefore, for our later convenience we introduce an $a d$ hoc fugacity $z$ and change the last rule as follows: for $i \geq j$ from the lattice site $(i, j)$ random walker can drop to the surface $(i+1,0)$ with the weight $z$.

The position of the random walker in lattice path will be denoted by the vector $|j\rangle$ in which $j$ is the height relative to the horizontal plane which is a number between 0 and $\infty$. These vectors have the following properties

$$
\begin{aligned}
& |j\rangle_{k}=\delta_{j, k} \quad \text { for } j, k=0,1, \ldots, \infty, \\
& \left\langle j \mid j^{\prime}\right\rangle=\delta_{j, j^{\prime}} \quad \text { for } j, j^{\prime}=0,1, \ldots, \infty, \\
& \sum_{j=0}^{\infty}|j\rangle\langle j|=\mathcal{I}
\end{aligned}
$$

in which $\mathcal{I}$ is an infinite-dimensional identity matrix. We assume that the random walker starts from the height $|j\rangle$ in which $j=0,1, \ldots, \infty$. After taking $t-1$ steps the random walker can get to the lattice site $\left(t-1, j^{\prime}\right)$ in which $j^{\prime}=j, j+1, \ldots, j+t-1$. There are different paths to get to the lattice site $\left(t-1, j^{\prime}\right)$. Each of these paths has its own weight. We now calculate the weight of a given path $\mathbf{p}$ as follow
$W_{\mathbf{p}}=w^{i}\left[\prod_{i=1}^{t-1} w^{\mathrm{step}}\left(e_{i}\right)\right] w^{f}$

in which $w^{i}$ and $w^{f}$ are the weights of the start and end points and $w^{\text {step }}\left(e_{i}\right)$ is the weight of the $i$ 'th step in the path. We know that the transfer matrix updates the state of the random walker hence according to the rules of the steps in the lattice path and their weights, the transfer matrix corresponding to this lattice path can be written as follow

$C|j\rangle=z|0\rangle+|j+1\rangle$.

The matrix representation of the transfer matrix $C$ is

$$
C=\left(\begin{array}{cccccc}
z & z & z & z & & \cdots \\
1 & 0 & 0 & 0 & 0 & \cdots \\
0 & 1 & 0 & 0 & 0 & \cdots \\
0 & 0 & 1 & 0 & 0 & \cdots \\
\vdots & \vdots & \vdots & \vdots & &
\end{array}\right) .
$$

\section{The partition function of the lattice path}

As we mentioned the random walker can start from the any height upper than the origin $|j\rangle$ in which $j=0,1, \ldots, \infty$. We have also assumed that the total number of steps is $t-1$. After taking these steps the random walker can get to the lattice site $\left(t-1, j^{\prime}\right)$ where $j^{\prime}=j, j+1, \ldots, j+t-1$ through different paths. Each of these paths has its own weight. The partition function of the walk model is the sum of the unnormalized weights of different paths consisting of $t-1$ steps that start from different heights $|j\rangle$ and get to the different heights $\left|j^{\prime}\right\rangle$ where $j=0,1, \ldots, \infty$ and $j^{\prime}=j, j+1, \ldots, j+t-1$. To obtain the partition function of the lattice path, we calculate the sum of the weights of all paths that start from the height $|j\rangle$ and, according to the mentioned rules, after $t-1$ successive steps get to the height $\left|j^{\prime}\right\rangle$. This sum is given by the following equation

$$
Z_{j, j^{\prime}}=w^{i}\left\langle j^{\prime}\left|C^{t-1}\right| j\right\rangle w^{f}
$$

in which $Z_{j, j^{\prime}}$ is the sum of unnormalized weights of different paths that start from the lattice site $(0, j)$ and after taking $t-1$ steps get to the lattice site $\left(t-1, j^{\prime}\right)$. According to the mentioned rules the weight of the start and end points for each path that starts from the height $|j\rangle$ and ends at the height $\left|j^{\prime}\right\rangle$ are given by

$$
w^{i}=q^{j}, \quad w^{f}=\left(\begin{array}{l}
j^{\prime} \\
j
\end{array}\right) \frac{1}{p^{j^{\prime}}} .
$$

Using the above equations the $Z_{j, j^{\prime}}$ can be written as 
$Z_{j, j^{\prime}}=\left(\begin{array}{c}j^{\prime} \\ j\end{array}\right) \frac{q^{j}}{p^{j^{\prime}}}\left\langle j^{\prime}\left|C^{t-1}\right| j\right\rangle$.

Considering that the lattice path can start from different height $|j\rangle$ in which $j=0,1, \ldots, \infty$ and end at different height $\left|j^{\prime}\right\rangle$ where $j^{\prime}=j, j+1, \ldots, j+t-1$ then the partition function of the lattice path can be written as

$Z=\sum_{j=0}^{\infty} \sum_{j^{\prime}=j}^{j+t-1}\left(\begin{array}{l}j^{\prime} \\ j\end{array}\right) \frac{q^{j}}{p^{\prime}}\left\langle j^{\prime}\left|C^{t-1}\right| j\right\rangle$.

Using (15) the partition function of the lattice path is given by the following relation

$Z_{t}(p, q, z)=\sum_{j=0}^{\infty} \sum_{j^{\prime}=j}^{j+t-1}\left(\begin{array}{c}j^{\prime} \\ j\end{array}\right) \frac{q^{j}}{p^{j^{\prime}}}\left(\sum_{k=0}^{t-2} z(z+1)^{t-k-2} \delta_{j^{\prime}, k}+\delta_{j^{\prime}, j+t-1}\right)$.

Hence, the partition function of the lattice path can be rewritten as

$$
\begin{aligned}
Z_{t}(p, q, z)= & \sum_{j=0}^{\infty} \sum_{j^{\prime}=j}^{j+t-1}\left(\begin{array}{c}
j^{\prime} \\
j
\end{array}\right) \frac{q^{j}}{p^{j^{\prime}}} z(z+1)^{t-j^{\prime}-2} \\
& +\sum_{j=0}^{\infty}\left(\begin{array}{c}
j+t-1 \\
j
\end{array}\right) \frac{q^{j}}{p^{j+t-1}} .
\end{aligned}
$$

Using Newton's binomial expansion the above equation can be rewritten as follows

$Z_{t}(p, q, z)=\sum_{j=0}^{\infty} \sum_{j^{\prime}=j}^{j+t-1}\left(\begin{array}{l}j^{\prime} \\ j\end{array}\right) \frac{q^{j}}{p^{j^{\prime}}} z(z+1)^{t-j^{\prime}-2}+\frac{1}{p^{t-1}}\left(1-\frac{q}{p}\right)^{-t}$.

Note that all parameters in the summand are non-negative thus using Tonelli's theorem we can interchange the summations, as $\left(\sum_{j=0}^{\infty} \sum_{j^{\prime}=j}^{t-1}\right) \equiv\left(\sum_{j^{\prime}=0}^{\infty} \sum_{j=0}^{j^{\prime}}\right)$. Hence, the partition function of the lattice path can be written as

$Z_{t}(p, q, z)=\sum_{j^{\prime}=0}^{\infty} \sum_{j=0}^{j^{\prime}}\left(\begin{array}{l}j^{\prime} \\ j\end{array}\right) \frac{q^{j}}{p^{j^{\prime}}} z(z+1)^{t-j^{\prime}-2}+\frac{1}{p^{t-1}}\left(1-\frac{q}{p}\right)^{-t}$.

We are interested in the partition function of the original walk model in the special case, that after taking $t-1$ successive steps, the random walker has taken a certain number of upward steps. We study the case in which after $t-1$ successive steps, the random walker can be at the heights between 0 and $t-M$ where $M \leq t$. To find the partition function of the model in this case, let us have a closer look at the role of the fugacity $z$. The weight associated with a horizontal or downward movement is proportional to $z$; therefore, the coefficient of $z^{M-1}$ in (41) is equal to the partition function of the walk model which consists of at most $t-M$ upward steps. The result is

$(z+1)^{t-j^{\prime}-2}=\sum_{i=0}^{t-j^{\prime}-2}\left(\begin{array}{c}t-j^{\prime}-2 \\ i\end{array}\right) z^{i}$

Using the above equation, the coefficient of the $z^{M-1}$ can be easily calculated as follows

$$
Z_{t, M}(p, q)=\sum_{j^{\prime}=0}^{t-M} \sum_{j=0}^{j^{\prime}}\left(\begin{array}{c}
j^{\prime} \\
j
\end{array}\right)\left(\begin{array}{c}
t-j^{\prime}-2 \\
M-2
\end{array}\right) \frac{q^{j}}{p^{j^{\prime}}} .
$$

One can interpret this partition function as the sum of the weights of all paths that have the length $t-1$ which contain $t-M$ upward steps (or equivalently $M-1$ horizontal and downward steps).

\section{The phase behavior of the lattice path in the thermodynamic limit}

As a relevant quantity, one can investigate the mean height of the random walker. The probability that the paths who starts from the height $|j\rangle$, and after $t-1$ successive steps according to the rules of the lattice path end at the height $\left|j^{\prime}\right\rangle$, is given by

$$
P_{t, M}\left(j, j^{\prime}\right)=\frac{1}{Z_{t, M}(p)} \frac{q^{j}}{p^{j^{\prime}}}\left(\begin{array}{c}
j^{\prime} \\
j
\end{array}\right)\left(\begin{array}{c}
t-j^{\prime}-2 \\
M-2
\end{array}\right) .
$$

Hence the average height of all possible paths in the lattice path is

$$
\langle h\rangle=\sum_{j^{\prime}=0}^{t-M} \sum_{j=0}^{j^{\prime}} j^{\prime} P_{t, M}\left(j, j^{\prime}\right) .
$$

It should be noted that in the above equation the arrangement of the index has been changed with respect to the Tonelli theorem. According to (42), the average of the height in lattice path is given by the following relation

$$
\langle h\rangle=-p \frac{\partial \ln Z_{t, M}(p)}{\partial p} .
$$

In the thermodynamic limit due to the behavior of the partition function of the lattice path, it turns out that the mean height of the random walker is given by

$$
\langle h\rangle=\left\{\begin{array}{ll}
t\left(1-\rho-\frac{p}{1+q-p} \rho\right) & \text { for } p-q<1-\rho(1+q) \\
\frac{p}{p-(1-\rho)(1+q)}-1 & \text { for } p-q>1-\rho(1+q)
\end{array} .\right.
$$

As can be seen in the thermodynamic $t \rightarrow \infty$, there is a phase transition from a phase in which the mean height of the random walker is of order $t$ to another phase where it is 
$(1-\rho)(1+q) /[p-(1-\rho)(1+q)]$. If $q=0$ the results are exactly those obtained in [11].

\section{Concluding remarks}

In this paper, we have introduced a two-species drivendiffusive model of classical particles defined on a onedimensional lattice with periodic boundary condition which can be mapped onto a zero-range process. The canonical partition function of the model is calculated and phase behavior of this model is investigated. After calculating the grand canonical partition function, the critical fugacity is obtained at which the model undergoes a firstorder phase transition. The density profile of the model is calculated exactly and the spatial correlations of the model are obtained in terms of 1-point correlation function. We have introduced a two-dimensional walk model in which the random walker, in contrast with the lattice path introduced in [11], can start from any height upper than the origin and that the end point of the lattice path can be at any height upper than the start point. This type of lattice path is introduced in [19]. The partition function of the lattice path is calculated using the transfer matrix method. Comparing this partition function with that of the drivendiffusive model we have shown that these two model are equivalent. It should be noted that the walk model introduced in [11] and the one introduced in present work can be mapped onto zero-range process.

Open Access This article is distributed under the terms of the Creative Commons Attribution 4.0 International License (http://crea tivecommons.org/licenses/by/4.0/), which permits unrestricted use, distribution, and reproduction in any medium, provided you give appropriate credit to the original author(s) and the source, provide a link to the Creative Commons license, and indicate if changes were made.

\section{References}

1. Lee, H.-W., Popkov, V., Kim, D.: Two-way traffic flow: exactly solvable model of traffic jam. J. Phys. A Math. Gen. 30, 8497 (1997)
2. Fouladvand, M.E., Lee, H.-W.: An exactly solvable two-way traffic model with ordered sequential update. Phys. Rev. E. 60, 6465 (1999)

3. Mallick, K.: Shocks in the asymmetry exclusion model with an impurity. J. Phys. A Math. Gen. 29, 5375 (1996)

4. Masharian, S.R., Jafarpour, F.H.: A heterogeneous zero-range process related to a two-dimensional walk model. Int. J. Mod. Phys. B 26(09) (2011)

5. Basu, U., Mohanty, P.K.: Spatial correlations in exclusion models corresponding to the zero-range process. J. Stat. Mech. L03006 (2010)

6. Jafarpor, F.H.: The application of the Yang-Lee theory to study a phase transition in a non-equilibrium system. J. Stat. Phys. 113, $112(2003)$

7. Arndt, P.F.: Yang-Lee theory for a nonequilibrium phase transition. Phys. Rev. Lett 84, 814 (2000)

8. Brak, R., de Gier, J., Rittenberg, V.: Nonequilibrium stationary states and equilibrium models with long range interactions. J. Phys. A Math. Gen. 37, 4303 (2004)

9. Brak, R., Essam, J.W.: Asymmetric exclusion model and weighted lattice paths. J. Phys. A Math. Gen. 37, 4183 (2004)

10. Blythe, R.A., Janke, W., Johnston, D.A., Kenna, R.: Continued fractions and the partially asymmetric exclusion process. J. Phys. A Math. Theor. 42, 325002 (2009)

11. Jafarpour, F.H.: Bose-Einstein condensation and a two-dimensional walk model. Phys. Rev. E 83, 041112 (2011)

12. Zeraati, S., Jafarpour, F.H.: Multiple-transit paths and density correlation functions in partially asymmetric simple exclusion process. Phys. Rev. E 83, 041133 (2010)

13. Evans, M.R., Hanney, T.: Nonequilibrium statistical mechanics of the zero-range process and related models. J. Phys. A Math. Gen. 38, R195 (2005)

14. Evans, M.R.: Phase transitions in one-dimensional nonequilibrium systems. Braz. J. Phys. 30, 42 (2000)

15. Evans, M.R.: Bose-Einstein condensation in disordered exclusion models and relation to traffic flow. Europhys. Lett. 36, 13 (1996)

16. Schtz, G.M., Ramaswamy, R., Barma, M.: Pairwise balance and invariant measures for generalized exclusion processes. J. Phys. A Math. Gen. 29, 837 (1995)

17. Mohammad Ghadermazi., Farhad H Jafarpour.: A new family of exactly solvable disordered reaction-diffusion systems. J. Stat. Mech. P09023 (2013)

18. Hinrichsen, H., Sandow, S., Peschel, I.: On matrix product ground states for reaction-diffusion models. J. Phys. A Math. Gen. 29, 2643-2649 (1996)

19. Brak, R., Essam, J.W.: Asymmetric exclusion model and weighted lattice paths. J. Phys. A Math. Gen 37, 14 (2004) 